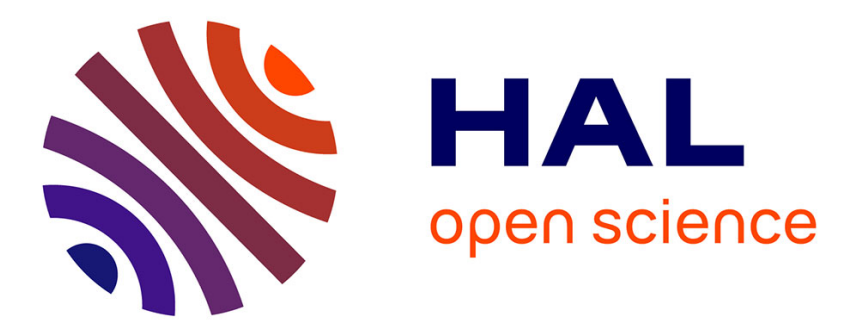

\title{
On the amplitude modulation and localization phenomena in interactive buckling problems
}

Angelo Luongo

\section{To cite this version:}

Angelo Luongo. On the amplitude modulation and localization phenomena in interactive buckling problems. International Journal of Solids and Structures, 1991, 27 (15), pp.1943-1954. hal-00803205

\section{HAL Id: hal-00803205 \\ https://hal.science/hal-00803205}

Submitted on 21 Mar 2013

HAL is a multi-disciplinary open access archive for the deposit and dissemination of scientific research documents, whether they are published or not. The documents may come from teaching and research institutions in France or abroad, or from public or private research centers.
L'archive ouverte pluridisciplinaire HAL, est destinée au dépôt et à la diffusion de documents scientifiques de niveau recherche, publiés ou non, émanant des établissements d'enseignement et de recherche français ou étrangers, des laboratoires publics ou privés. 


\title{
ON THE AMPLITUDE MODULATION AND LOCALIZATION PHENOMENA IN INTERACTIVE BUCKLING PROBLEMS
}

\author{
A. LuONGo \\ Dipartimento di Ingegneria delle Strutture. Universita di L'Aquila. Monteluco di Roio. \\ 67040 L'Aquila, Italy
}

(Receited 7 April 1990) : in revised form 8 Octoher 1991$)$

\begin{abstract}
The postcritical behavior of a 3D system of elastically restrained beams. which can manifest overall and local modes, is analyzed. The secondary bifurcation in the overall postcritical range is studied. By applying the multiple scale perturbation method it is found that the amplitude modulation phenomenon is governed by a differential equation of the second order. An approximate anilytical expression of the amplitude modulating function is obtained by the WKB asymptotic method. The occurrence of a turning point which is responsible for the strong localization is revealed. Close analogies with lecalization of vibrations in imperfect systems are highlighted.
\end{abstract}

\section{INTRODUCTION}

Systems which exhibit a great number of nearly simultaneous modes are characterized in the postcritical range by buckling patterns that strongly depend on the intensity of the load. In particular, the deformation can localize in one or more regions of limited size, in contrast with the periodic character of critical modes. The phenomenon is known as localization and it has been studied both experimentally (Moxham. 1971) and theoretically. Tvergatard and Needleman (1980) have made reference to diverse models of imperfect systems, with nonlincarities of the softening type, observing that the localization is the conseyuence of a bifurcation which occurs immediately after altainment of the limit point, on the unstable branch of the equilibrium path. A linear combination of the primary periodic mode and of the bifurcattion mode can produce a preferential growth of one of the waves and thus explain the localization tendency. Poticr-Ferry (1987) has studied the postcritical behavior of a beam on a nonlinear elastic soil, showing that, at the restraints, the hardening nonlincarities can produce limit layers wherein the amplitude of the buckling is modulated. The localization occurs in the presence of softening nonlinearities and is attributable to the high modal density rather than to the insurgence of secondary bifurcation. Benito and Sridharan (1985), Sridharan and Ali (1985), Byskov (1988), Byskov et al. (1989) and Luongo and Pignataro (1988) have analyzed the problems of interaction among local and overall simultancous modes of assemblies of compressed plates; in particular, in the last paper, it is shown that the lincar combination of several critical modes gives rise to localized buckling.

In recent years, in parallel with these studies, research has been pertormed on problems of localization of the oscillations in imperfect structures at high modal densities, such as systems formed by a great number of weakly coupled substructures [see, for instance, Pierre and Cha (1989) and Cornwell and Bendinksen (1989)]. In such systems small local variations in stiffness and/or mass destroy the periodic character of the vibration modes which localize in one or more region of the structure. The extent of the localization is proportional to the ratio between the amplitude of the imperfections and the degree of coupling among substructures. Hence localization increases with modal density. The phenomenon can be explained on the basis of a linear theory and is closely bound up with the insurgence of turning points in the solution, as demonstrated by Luongo (1988).

An in-depth study of the analogies between the two problems lies outside the scope of this paper. What is done here is to analyze the static phenomenon with the help of a specific model. the aim being to demonstrate that, as in the dynamic problem. the localization mechanism calls for satisfaction of two requisites: (a) that the system should be of high modal density, and (b) that there should be present a "structural irregularity" in the broad sense. The example given concerns a 3D beam system on elastic soil which exhibits an 


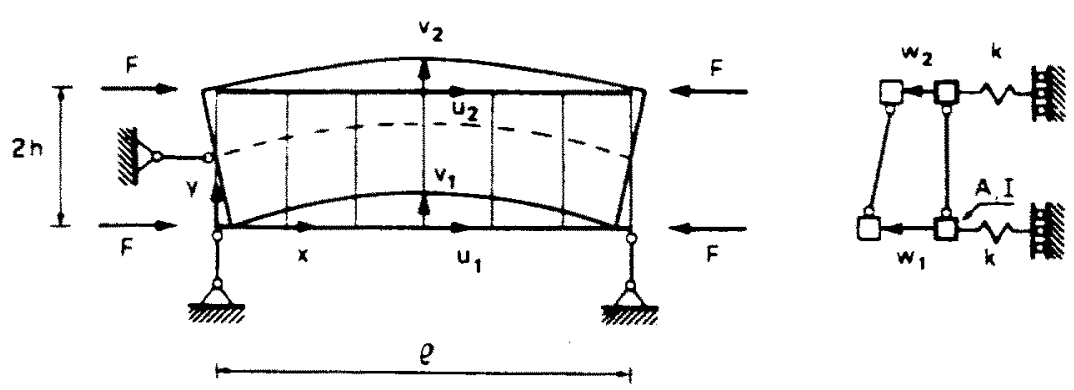

Fig. 1. 3D model of elastically restrained beams.

overall mode and several local modes that affect a single beam. The behavior of the model is similar to the plate assembly studied in the paper by Luongo and Pignataro (1988). However, here a secondary bifurcation along a nonlinear equilibrium path is dealt with. It is assumed that the second bifurcation is close enough to the first to linearize the problem in the amplitude of the overall mode which is taken as being the control parameter. The equation that governs the secondary bifurcation has slowly and weakly varying coefficients so that the efrect of the primary bifurcation reflects in a "structural defect" which, together with the high modal density, is responsible for the localization. Through the use of the multiple scale perturbation method it is possible to determine an equation in the (variable) amplitude of the secondary bifurcation mode; as in the dynamic case. this leads to a turning point problem which is solved by the WKB method. It is shown that if the modal density is sulficiently high, the amplitude of the mode lends rapidly to zero outside a small region in which it is weakly variable; the local buckling is thus localized.

Only the hifurcation analysis is performed here and no information is obtained about the local postcritical behavior of the system. Consequently, even though the bifurcation mode may be highly localised in chatracter, it does not mean that the structure would collapse in such a localized mode, since geometric nonlinearities could reduce the effects of the amplitude modulation in the postbifurcation range

\section{STRUCTURAL MODEL AND POSTCRITICAL BIFURCATION}

Let us consider the model in Fig. I, consisting of two elastic beams connected by bars which are rigid in the $\mathrm{xy}$ plane and infinitely flexible out-of-plane. The beams are continuously restrained by elastic springs orthogonal to the plane and are compressed by forces $F$. The structure is simply supported.

Two instability forms can occur: one of overall type, in which the structure behaves as a single shear-indelormable built-up beam, the other of local type, in which each beam buckles independently out-of-plane, restrained by the springs. From this point of view the behavior of the structure is similar to that of compressed thin-walled beams that can exhibit either overall instability (Eulerian or llexural-torsional) or local instability of the component plates.

In the system concerned overall instability occurs when $F$ is equal to the Eulerian critical load

$$
F_{\mathrm{c}}=\pi^{2} E A h^{2} / l^{2}
$$

where $/$ is the length. $E$ the elastic modulus and $A$ the cross-sectional area of each beam, whose dimensions are assumed to be negligible compared with the haltheight $h ; h / l \ll 1$ is also assumed. The overall critical mode associated with the load $F_{\mathrm{c}}$ is

$$
\begin{aligned}
& u_{1.2}(x)= \pm v_{0} \pi(h / l) \cos (\pi x / l) \\
& r_{1.2}(x)=v_{0} \sin (\pi x / l) \\
& u_{1.2}(x)=0
\end{aligned}
$$


where indexes 1 and 2 refer to the bottom and top beams, respectively, and $c_{0}$ is the midspan defiection.

The local instability is governed by the equation of the beam on elastic soil

$$
E / w^{\prime \prime \prime}+\left(N w^{\prime}\right)^{\prime}+k w=0
$$

where the apex denotes differentiation with respect to $x, I$ is the inertia moment of the beam with respect to the centroidal axis parallel to $y, k$ is the stiffness of the springs and $N=F=$ const the axial force : moreover $u \equiv w_{1,2}$. Assuming that the beams are simply supported at the ends then $w=w^{\prime \prime}=0$ in $x=0 . l$, so that eqn (3) admits the eigenfunction

$$
w(x)=w_{0} \sin (n \pi x / l)
$$

and the eigenvalue

$$
F_{l}=\left(n^{2} \beta^{2}+\frac{1}{n^{2} \beta^{2}}\right) \sqrt{k E l}
$$

where

$$
\beta=(\pi / l) \sqrt[4]{E I / k}
$$

has been posed. In eqn (4), $n$ is an integer which must be determined by making $F_{l}$ a minimum, so it depends on the dimensionless parameter $\beta$. If the wavelength of the local mode is small compared with the span of the beam, i.c. $n$ is large (c.g. $n \geqslant 20$ ), a large number of nearly simultaneous modes exist, corresponding to loads close to

$$
F_{l}=2 \sqrt{k E l}
$$

obtained by posing $n=1 / \beta$ in eqn (5). In this case the parameter $\beta$ assumes the meaning of the ratio between the wavelengths of the local and overall modes, and by hypothesis is a small quantity. Besides it is easy to see from eqn (5) that $\beta$ is a measure of the modal density of the system, that increases for decreasing values of the parameter.

In the paper by Luongo and Pignataro (1988), the postcritical behavior of thin-walled compressed beams was analyzed assuming that the overall and local critical loads are coincident. In particular, the nonlinear analysis of the multiple bifurcation has revealed the occurrence of localization phenomena. Here we consider the case in which $F_{1}$ is slightly higher than $F_{\mathrm{c}}$ and limit ourselves to determining the secondary bifurcation point and the associated critical mode that occurs for a load larger than the critical one. The (stable) bifurcated path corresponding to the primary bifurcation is described by the parabola

$$
F=F_{\mathrm{c}}\left(1+\frac{\pi^{2}}{8} \frac{v_{0}^{2}}{l^{2}}\right)
$$

along which, to within terms proportional to $t_{u}^{\prime}$, the deflection of the system is given by eqns (2). In the postcritical range, due to this displacement ficld, the normal force acting on the two beams is varied by

$$
\Delta N_{1,2}=\mp E A u_{1,2}^{\prime}= \pm F_{c}\left(c_{0} / h\right) \sin (\pi x / l)
$$

Therefore the resultant force is $N_{1,2}=F+\Delta N_{1,2}$ and so taking account of eqn (8), it is 


$$
N_{1.2}=F_{c}\left[1 \pm\left(c_{0} / h\right) \sin \left(\pi x_{i} l\right)\right]
$$

where quadratic terms in $t_{1}$, have been omitted.

By assuming $r_{0}>0$. the secondary bifurcation occurs when the critical equilibrium is reached in the bottom beam. which is more compressed than the top beam. The condition of critical equilibrium must be formulated referring to the varied configuration of the beam. which is compressed and bent in the ry plane, and therefore concerns the stability of an arch for out-of-plane displacements. If the cross-section is symmetric with respect to the $y$ axis, the critical mode consists of a lateral deflection and twisting of the beam. Nevertheless. in the problem under consideration, it is possible to show that the angle of twist is small, of order $c_{11} h$. and its influence in the force equilibrium is small. of order $\left(c_{1} h\right)^{2}$. so that it can be neglected. Therefore, the bifurcation condition corrected to first order is expressed in the single variable $"$ and is still given by eqn (3). except for the normal force which is now a function of $x$ according to eqn (10). By referring to the bottom beam and omitting index 1 , from eqn (10) we obtain

$$
\left(N i^{\prime}\right)^{\prime}=F_{c} u^{\prime \prime}+F_{v}\left(c_{0} / h\right)\left[u^{\prime} \sin (\pi x / l)\right]^{\prime}
$$

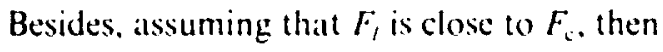

$$
F_{l}=F_{c}(1+i)
$$

with $0<\because \ll 1$. and ecpn (3) can be put in the form

$$
\left[: w^{\prime \prime \prime \prime}+F_{1} w^{\prime \prime}+k w+F_{s} i\left[\left(v_{0} / h\right) \sin (\pi x / l)-\gamma_{1}\right] w^{\prime \prime} ; .\right.
$$

Equation (13), with associated boundary conditions, is a linear cigenvalue problem in $e_{0} / h$ and in the eigenfunction $n(x)$. The first three terms constitute the equation of the beam on elastic soil subjected to the locial critical load. Of the last two terms, which are small compared with the others. the first one represents the effect of the primary bifurcation (which is the caluse of the variable coeflicients in the equation) and the second the effect of the cluseness of two critical loads. These terms can be considered as perturbitions of the equation of the beam on elastic soil and represent, in one sense, "defects" of the geometric stiffiness of the system. It will be seen suceessively that the perturbations and the high modal density are responsible for the localization.

In the following sections eqn (13) is solved by applying first the Galerkin technique and then the multiple scale perturbation method.

\section{GALERKIN SOLUTION}

The unknown function $w(x)$ in eqn (13) is expressed by the series expansion

$$
w=\sum_{1=1}^{\prime}(c, j) w
$$

where $u_{t}=\sin (j \pi x / l)$ are the eigenfunctions of eqn (3), in which $N$ is a constant. Note that. for convenience. coefficients $c$, halve been divided by $j$.

By applying the standard Galerkin technique, and accounting for the orthogonality properties of the eigenfunctions $w^{\prime}$, the following algebraic eigenvalue problem, of infinite dimension, is obtained

$$
\sum_{i=1}^{\prime} c_{i}\left[\left(\mu+\sigma_{1}\right) \dot{\delta}_{1 \prime}-2 i \chi_{1 \prime}\right]=0 \quad(i=1,2 \ldots)
$$

Here $\delta_{1}$, is the Kronecker symbol and 


$$
\begin{aligned}
\chi_{1 \prime} & =\int_{0}^{1} \sin \pi \xi \cos i \pi j \cos j \pi j \mathrm{~d} \xi \\
& \approx \frac{1}{\pi\left[(i-j)^{2}-1\right]} \quad(i \pm j \text { even })
\end{aligned}
$$

where $i \gg 1, j \gg 1$ have been considered. Moreover, the following positions have been introduced

$$
\lambda=\frac{1}{\beta^{2}} \frac{v_{10}}{h}, \mu=\frac{i}{\beta^{2}}
$$

together with

$$
\sigma_{i}=\frac{F_{i}-F_{i}}{\beta^{2} F_{\mathrm{c}}} \approx\left\{\frac{1}{2}\left[\left(\frac{j}{n}\right)^{2}+\left(\frac{n}{j}\right)^{2}\right]-1\right\} n^{2} .
$$

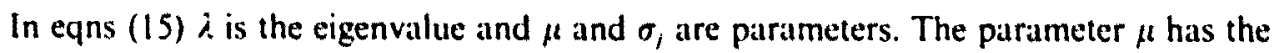
meaning of the ratio between a mealsure of the "imperfection" $\gamma$ and a measure of the modal density: the eigenvalue problem depends on this ratio, in analogy with problems of dynamic localization. Parameters $\sigma_{i}$, instead, account for the small differences between the higher local critical loads $F$, and the lower critical load $F_{t}$.

By considering only $M$ terms in the series (14), eyns (15) become an $M \times M$ eigenvalue problem. Since matrix $\left[\chi_{11}\right]$ is symmetrical and positive definite, eqns (15) admit $M$ real solutions with $\lambda,>0$ corresponding to as many secondary bifurcations. Here we are interested in the minimum $\lambda_{2}$, i.e. $\lambda_{b}$ which corresponds to the first bifurcation. By numerically solving the eigenvalue problem the following results are obtained.

(a) Only the harmonics with wavenumber $j$ which is close to $n$ give significant contributions to the solution. In fact, coeflicients $c_{j}$ rapidly decrease for increasing values of the differences $|j-n|$.

(b) For a fixed $n$ and an increasing number $M$ of harmonics, $\lambda_{b}$ very rapidly converges to an asymptotic value which depends on $\mu$. Table I shows, for various $\mu$, the $\lambda_{b} / \mu$ ratios obtained considering $j=n, n \pm 2, \ldots, n \pm 2 m$ in the eigenvalue problem. It is seen that the rate of convergence decreases for increasing $\mu$; however, in the range examined, $M=2 m+1=11$ harmonics are sufficient to furnish a good approximation of the eigenvalue. Similar results are obtained for the corresponding eigenvector.

(c) The eigenvalue $\dot{i}_{b}$ is practically independent of $n$. In fact, by varying $n$ from 25 to 100 . $\lambda_{b}$ varies by only a few units per thousand. This result can be explained by the fact that coefficients $\chi_{i j}$ do not depend on $n$ [see eqn (16)] while parameters $\sigma$, are weakly dependent on $n$ for $j$ close to $n$, i.e. in correspondence with the most important harmonic components. In fact, by posing $j=n \pm k$, with $k \ll n$, eqn (18) furnishes $\sigma_{1}=2 k^{2}+O(k / n)$.

Table 1. Convergente of the minimum eigenvalue $(n=25)$

\begin{tabular}{rcccc}
\hline & \multicolumn{4}{c}{$\lambda_{n} / \mu$} \\
\cline { 2 - 5 }$n$ & $m=2$ & $m=3$ & $m=5$ & $m=7$ \\
\hline 10 & 1.3251 & 1.3251 & 1.3251 & 1.3251 \\
100 & 1.1050 & 1.1040 & 1.1040 & 1.1040 \\
200 & 1.0762 & 1.0730 & 1.0728 & 1.0728 \\
\hline
\end{tabular}




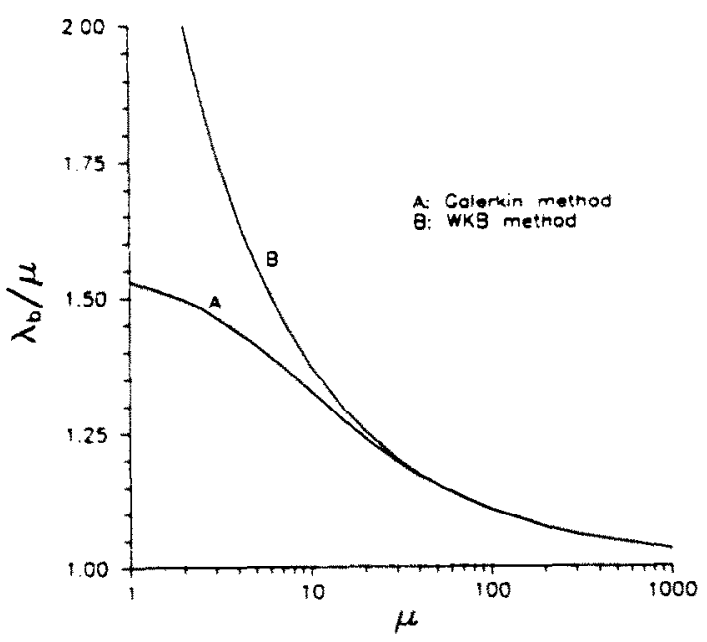

Fig. $2 . \lambda_{b} / \mu$ ratios vs $\mu$ by different methods.

(d) For increasing $\mu$, the $i_{h} / \mu$ ratio decreases and tends towards 1 when $\mu \rightarrow \infty$ (Fig. 2 . curve A), i.e. for very high modal density. If this asymptotic result is used, $c_{0} / h=\gamma$ is obtained from eqn $(17)$, then the bifurcation load $F_{b}$ on the postbuckling equilibrium path follows from eqn (8):

$$
F_{b}=F_{c}\left(1+\begin{array}{l}
\pi^{2} h^{2} \\
8 l^{2}
\end{array}\right)
$$

Since $(h / l)^{2} \ll 1, F_{\mathrm{h}}<F_{1}$ from comparison with eyn (12). The secondary bifurcation therefore occurs at a lower load than that which would oceur in the absence of an interaction, with a consequent reduction in the stable part of the bifurcated path.

(e) At the bifurcation, the local deflection of the beam is represented by an oscillating function with strong modulated amplitude (Fig. 3). The modulation depends only on the value of the parameter $\mu$, not on the number $n$ of the halliwaves of the local critical mode, as it appears by comparing curves with the same values of $\mu$.

\section{AMPLITUDE MODULATION OF THE LOCAL BUCKLING: GOVERNING EQUATION}

The analysis performed in the previous section necessarily requires numerical calculus to solve an eigenvalue problem. Besides the solution is represented by a linear combination of functions, so it is not very suitable for concisely describing the phenomenon. For these reasons it is better to follow another procedure, similar to that adupted by Potier-Ferry (1987) for a nonlinear differential equation with constant coefficients.

To this end it is convenient to render eqn (13) dimensionless by introducing the new variables

$$
\tilde{x}=\pi x /(\beta l), \quad \tilde{u}=w / T
$$

where $I$ is a length. In terms of dimensionless variables, remembering that $n=1 / \beta$, the local critical mode is of the type $\sin \bar{x}$ and the overall one is $\sin \beta . \bar{x}$. In terms of new variables. eqn (13) is written as

$$
\left.w^{\prime \prime \prime \prime}+2 w^{\prime \prime}+w^{*}=-2 \beta\right)^{2}\left[(i \sin \beta x-\mu) w^{\prime}\right]^{\prime}
$$

with the boundary conditions 

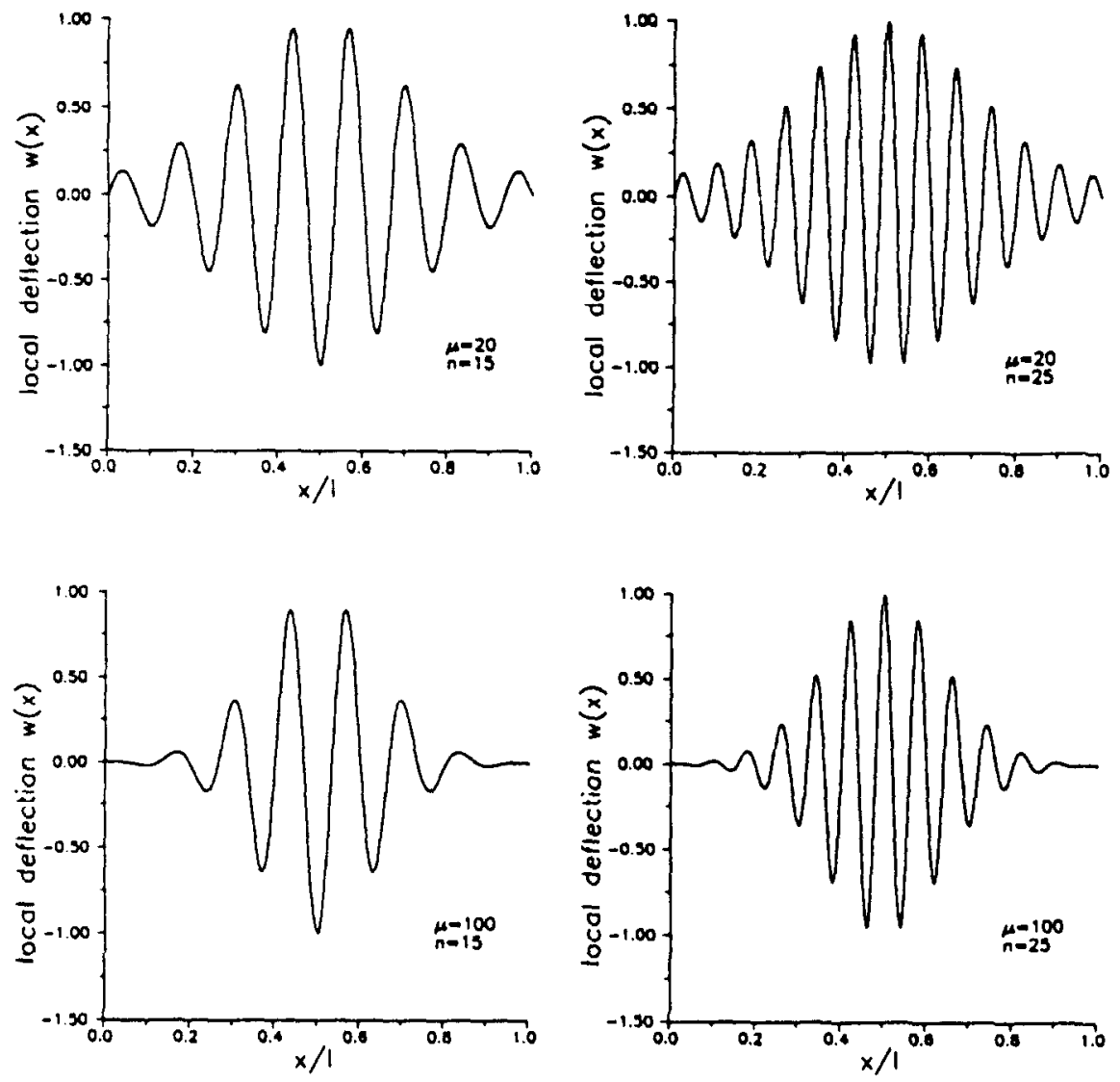

Fig. 3. Locill deflections when $\mu-20,100$ and $n=15,25$.

$$
w(0)=w^{\prime \prime}(0)=w(\pi / \beta)=w^{\prime \prime}(\pi / \beta)=0
$$

having omitted the tilde and hatving still denoted by an apex differentiation with respect to the dimensionless variable. Equations (6), (7) and (17) have also been taken into account and $y \ll 1$ has been considered.

The coeflicients of eqn (21) vary slowly and weakly. This suggests use of the multiple scale perturbation method (Nayfeh, 1973), which permits attainment of a differential equation in the variable amplitude of the mode. The unknown function is expanded in a $\beta$ series

$$
w=\beta w_{1}+\beta^{2} w_{2}+\beta^{3} w_{3}+O\left(\beta^{4}\right)
$$

and is assumed to depend on several variables $x_{0}, x_{1}, \ldots$ related to $x$ by equations

$$
x_{0}=x, \quad x_{1}=\beta x, \quad x_{2}=\beta^{2} x
$$

$x_{0}$ represents the fast variable, associated with the law of the local mode: $x_{1}$ the slow variable, associated with the law of the overall mode [and coefficients of eqn (21)] $; x_{2}$ is a slower variable. Using the chain rule the derivatives are transformed according to

$$
\begin{aligned}
& \mathrm{d}^{2} / \mathrm{d} x^{2}=D_{00}^{2}+2 \beta D_{01}^{2}+\beta^{2}\left(D_{11}^{2}+2 D_{02}^{2}\right)+\cdots \\
& \mathrm{d}^{4} / \mathrm{d} x^{4}=D_{0000}^{4}+4 \beta D_{0001}^{4}+\beta^{2}\left(6 D_{0011}^{4}+4 D_{0102}^{4}\right)+\cdots
\end{aligned}
$$

where the operator $D$ denotes differentiation with respect to the variables having the same 
indexes. By substituting eqn (23) in eqn (21) and taking into account eqns (25). the following perturbation equations are obtained:

$$
\begin{aligned}
& \beta: L\left(w_{1}\right)=0 \\
& \beta^{2}: L\left(w_{2}\right)=-4\left(D_{1001}^{4}+D_{i 1}^{2}\right) w_{1} \\
& \beta^{2}: L\left(w_{3}\right)=-\left(6 D_{111_{1}}^{+}+2 D_{i_{1}}^{i}\right) w_{1}-4\left(D_{1 w_{02}}^{+}+D_{10_{2}}^{*}\right) w_{1} \\
& -4\left(D_{i n+1}^{4}+D_{i 1}^{i}\right) \mu_{2}-2 D_{\mathrm{n}}\left[\left(i \sin x_{1}-\mu\right) D_{4} H_{1}\right]
\end{aligned}
$$

where $L=D_{0,10}^{4}+2 D_{00}^{2}+1$. In the same way the boundary conditions to be imposed in $x=0, \pi / \beta$, result in :

$$
\begin{aligned}
& \beta: w_{1}=0, \quad D_{i n}^{2} w_{1}=0 \\
& \beta^{2}: w_{2}=0 . \quad D_{b 0}^{2} w_{2}=-2 D_{i 1}^{2} w_{1}
\end{aligned}
$$

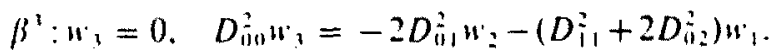

The solution to eyns (26a) and (27a) is

$$
w_{1}=a\left(x_{1}, x_{2}\right) \sin x_{10}
$$

It is therefore a sinusoidal function the amplitude of which is modulated on the slow scales. Note that in eqn (28) the phase was not introduced because in eqn (26) only even derivalives appear. Therefore, at the order $\beta^{\prime}$. no phase modulation exists but only amplitude modulation.

By substituting eqn (28) into eqn (26b). accounting for $\left(D_{0 w+11}^{4}+D_{b 1}^{+}\right) w_{1}=0$ and integratting, the following gencral solution is obtained

$$
u_{2}=\left[b\left(x_{1}, x_{2}\right) x_{0}+c\left(x_{1}, x_{2}\right)\right] \sin x_{0}
$$

where $b$ and $c$ are arbitrary lunctions of the slow variables $x_{1}, x_{2}$ : moreover

$$
h(0,0)=-D_{1} a(0,0), \quad h(\pi, \beta \pi)=-D_{1} a(\pi, \beta \pi)
$$

must hold good to satisfy boundary conditions (27b).

In order to make the asymptotic expansion (23) uniformly valid in the interval $0 \leqslant x_{1} \leqslant \pi / \beta, b\left(x_{1}, x_{2}\right)=0$ is assumed. In addition $c\left(x_{1}, x_{2}\right)=0$ is chosen, since the function $c \sin x_{0}$ repeats the $\beta$-order solution (28). In conclusion, the $\beta^{2}$-order equations yield $n_{2}=0$ together with $D_{1} a=0$ at the boundaries.

By replacing $w_{z}$ and $w_{2}$ in eqn (26c) and imposing the coefficients of $\sin x_{1}$ to be zero (solvability condition), it follows that

$$
\frac{d^{2} a}{d x_{i}^{2}}+\frac{1}{2}\left(i \sin x_{1}-\mu\right) a=0
$$

where $a=a\left(x_{1}\right)$ has been assumed. because the dependence on $x_{2}$ remains undetermined at this order. The differential equation (31), integrated with the boundary conditions

$$
\frac{\mathrm{d} a}{\mathrm{~d} x_{1}}(0)=0, \quad \frac{\mathrm{d} a}{\mathrm{~d} x_{1}}(\pi)=0
$$

permits the determination of the function $a\left(x_{1}\right)$, i.e. the law of amplitude modulation. 
Equations (31) and (32) constitute a second-order boundary value problem with variable coefficients. which can be put in the form

$$
\begin{gathered}
\frac{\mathrm{d}^{\prime} a}{\mathrm{~d} x_{i}^{2}}+f\left(x_{1}\right) a=0 \\
\frac{\mathrm{d} a}{\mathrm{~d} x_{1}}(0)=0, \quad \frac{\mathrm{d} a}{\mathrm{~d} x_{1}}(\pi / 2)=0
\end{gathered}
$$

where

$$
f\left(x_{1}\right)=\frac{1}{2}\left(j \cos x_{1}-\mu\right)
$$

Note that the origin of the abscissa $x_{1}$ has been shifted to the midspan and only symmetrical solutions, to which lower eigenvalues $\lambda_{b}$ correspond, are considered.

A preliminary numerical solution of the problem (33)-(34) has been performed. The solution has been obtained by a shooting method. by employing a Runge-Kulta method to solve the initial-value problem and the bisection procedure to satisfy the condition at the endpoint of the interval. Results obtained are in excellent accord with those of the Gallerkin solution. to within an error of some units per thousind, so they are not shown here. This analysis confirms the validity of the multiple scale perturbation approach and confirms that the phenomenon is in fact governed by the amplitude modulation differential eqn (33). In particular, by remembering eyns (20a) and (24b), it is seen that the amplitude modulating function $a\left(x_{1}\right)$ does not depend on $n=1 / \beta$, as previously found by the Galerkin analysis.

With the aim of furnishing a simple, analytical solution to the boundary value problem (3.3) (34), we first distinguish two casces:

(a) $i<\mu$ : becaluse $f\left(x_{1}\right)<0$ in the whole domain, the solution $a\left(x_{1}\right)$ is exponential;

(b) $i \geqslant \mu$ : there is a point $x_{1}=\Delta$ (turning or transition point) where $f(\Delta)=0$; becaltuse the function changes sign in $[0, \pi / 2]$ the solution is oscillating for $x_{1}<\Delta$ and exponential for $x_{1}>\Delta$.

However, in case (a) no solutions to the boundary value problem exist. In fact, due to $f^{\prime}\left(x_{1}\right)<0$, from eqns (33) and (34a) it follows that $\mathrm{d}^{2} a / \mathrm{d} x_{1}^{2}>0$ in the whole interval [if $a(0)>0]$, so that eqn (34b) cannot be satisfied.

The turning point problem, case (b), can instead be solved by applying the WKB perturbation method, provided $\mu$ assumes sufficiently large values. An application of the method has been presented in a paper by the author (Luongo, 1988) where details of the procedure are given.

The WKB solution reads:

$$
\begin{array}{lll}
a\left(x_{1}\right)=\left(f\left(x_{1}\right)\right)^{-1+4}\left[c_{1} \cos \left(\psi_{1}\left(x_{1}\right)+\pi / 4\right)+c_{2} \sin \left(\psi_{1}\left(x_{1}\right)+\pi / 4\right)\right] & \left(x_{1}<\Delta\right) \\
a\left(x_{1}\right)=\left(-f\left(x_{1}\right)\right) & { }^{1}\left[c_{1} \exp \psi_{2}\left(x_{1}\right)+\left\lfloor c_{2} \exp \left(-\psi_{2}\left(x_{1}\right)\right)\right]\right. & \left(x_{1}>\Delta\right)
\end{array}
$$

where $c_{1}$ and $c_{2}$ are arbitrary constants and

$$
\psi_{1}\left(x_{1}\right)=\int_{r_{1}}^{a} \sqrt{ } j(\xi) \mathrm{d} \xi . \quad \psi_{2}\left(x_{1}\right)=\int_{\Lambda}^{1} \sqrt{ }-f(\xi) \mathrm{d} \xi .
$$

In eqns (37) $\Delta=\Delta(\lambda)=\operatorname{arcos}(\mu / \lambda)$ is the abscissa of the turning point, which is unknown. because of its dependence on $\lambda$.

Equations (36) are singular at $x_{1}=\Delta$ so that they are not valid near the transition point. In this region there exists a transient condition that can be accurately described by the Airy functions. or equally by the Bessel functions, that are not reported here [see, for 
instance, Handhook of Mathematical Functions (1972)]. The solutions in the inner and outer regions are then matched by applying the matching principle (Nayfeh, 1973).

By imposing the boundary conditions (34) the following algebraic equations in the two arbitrary constants are obtained

$$
\left(\begin{array}{cc}
-\sin \left(\psi_{1}(0)+\pi / 4\right) & \cos \left(\psi_{1}(0)+\pi / 4\right) \\
x_{1} \exp \psi_{2}(\pi / 2) & -\left(x_{2} / 2\right) \exp \left(-\psi_{2}\left(\pi_{2} 2\right)\right)
\end{array}\right)\left\{\begin{array}{l}
c_{1} \\
c_{2}
\end{array}\right\}=\left\{\begin{array}{l}
0 \\
0
\end{array}\right\}
$$

where

$$
x_{1,2}=1 \pm \lambda / \sqrt{8 \mu^{3}}
$$

By requiring the determinant of the matrix of the coefficients to vanish the following characteristic equation is obtained

$$
\tan \left(\psi_{1}(0)+\pi / 4\right)=2\left(x_{1} / x_{2}\right) \exp \left(2 \psi_{2}(\pi / 2)\right)
$$

Equation (40) must be solved for the eigenvalue $\lambda$ accounting for the positions (37) and $\Delta=\Delta(\lambda)$. However, an approximate solution can be obtained, $\mu$ being large by hypothesis. By assuming $j=O(\mu)$ then $x_{1} / \alpha_{2}=O(1)$ and $\psi_{2}(\pi / 2)=O(\sqrt{\mu})$ so that eyn $(40)$ admits the approximate solution tan $\left(\psi_{1}(0)+\pi / 4\right)=\infty$, i.e.

$$
\begin{gathered}
\psi_{1}(0)=\pi(k-3 / 4) \quad(k=1,2, \ldots) \\
c_{1}=0, \quad c_{2}=1 .
\end{gathered}
$$

Note that equs (41) exictly satisfy only the condition of symmetry (38:1) at $x_{1}=0$, not the boundary condition (38b); however, they are a good approximation of the solution when $\psi_{:}(\pi / 2)$ is sufficiently large. Note also that the eigenvalue problem admits infinite solutions, each corresponding to a bifurcation.

Equation (4lat) can be easily solved in closed form for the cigenvalue $i$ if $f\left(x_{1}\right)$ is approximated by a parabola in the interval $[0, \Delta(\lambda)]$, with an error whose size decreases with decrease in $\Delta$, i.e. the greater the localization of the mode. By proceeding in this way we obtain from eq̣n (35)

$$
f\left(x_{1}\right)=\lambda\left(\Delta^{2}-x_{1}^{2}\right) / 4
$$

where

$$
\Delta^{2}=2(1-\mu / i)
$$

Introducing eqn (42) in eqn (37a) and integrating we obtain $\psi_{1}(0)=\pi / \sqrt{ } \cdot \Delta^{2} / 8:$ after replacing in eqn (41), in which $k=1$. and accounting for eqn (43) we finally get

$$
\lambda_{n}=\mu+!+\sqrt{\mu+1}
$$

where $1 / 2$ and $1 / 4$ can eventually be neglected with respect to $\mu$. Introducing eqn (44) in eqn (43) the abscissa of the turning point is determined as a function of $\mu$. Equation (44) is plotted in Fig. 2, curve B, and compared with the Galerkin solution [or, equally. with the numerical solution of eqns (33). (34)]. It is seen that the WKB approximation is very good when $\mu \geqslant 20$, despite the many assumptions made in the analysis. 


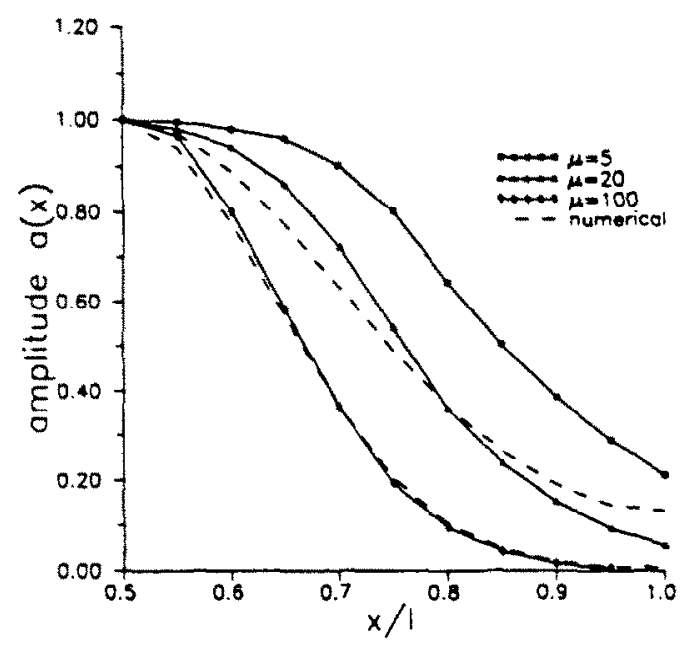

Fig. 4. Amplitude modulating functions: analytical and numerical solutions.

The curves $a\left(x_{1}\right)$ determined for different values of $\mu$ from eqns (36) and those obtained by numerically integrating eqns (33)-(34) are plotted in Fig. 4 vs the original abscissa $x$. When $\mu=20$, the differences between the two solutions are noticeable. because of the error associated with the boundary conditions at $x_{1}=\pi / 2$. However, when $\mu$ increases, the error rapidly tends to zero and the two curves approach each other. Obviously, a more accurate amalytical solution can be obtained if eqns (38) -(40) are solved exactly.

Figure 4 shows that the degree of localization increases with $\mu$, and it is rather high even for relatively small $\mu$. For example, for a ratio $F_{/} / F_{\mathrm{c}}=1.1$ and a local mode with wavenumber $n=10$, i.t. for a modal density that is not very high, it ensues that $y=0.1$, $\beta=0.1$ and so $\mu=10$ from eqn (17b). For the same ratio between the critical loads and higher modal density, e.g. $n=20$, then $\mu=40$ and the local deflection is strongly localized: the corresponding amplitude of the overall mode is obtained from eqns (17a) and (44): $v_{0} / h=0.11$. i.e. $l_{0} / h \approx \gamma$.

The "exact" amplitude modulating functions relative to $\mu=20,100$ are also reported in Fig. S. for comparison with the Gaterkin solution. It is apparent that they accurately describe the buckling patterns.

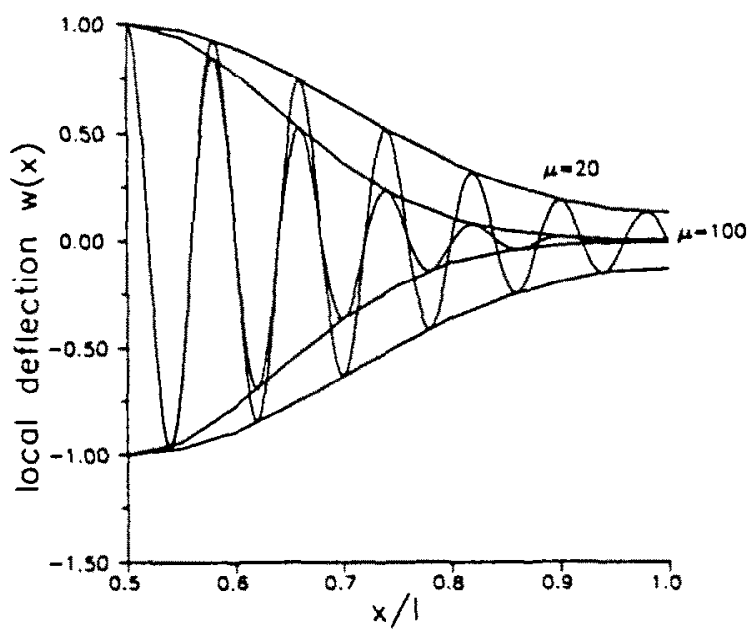

Fig. 5. Galerkin solutions (oscillating functions) and perturbative solutions (amplitude modulating functions). 
The postcritical behavior of a 3D system of elastically restrained beams, characterized by a high modal density, is analyzed. The point of secondary bifurcation associated with local instability is determined on the bifurcated branch corresponding to an overall instability mode. Analysis of the local buckling has highlighted amplitude modulation phenomena and localization due to two concomitant causes: (a) high modal density of the system. and (b) variable geometric stiffness due to the effect of primary bifurcation. The magnitude of the localization has been found to depend on the ratio involving the difference between two critical loads, which is assumed to be small. and a parameter which decreases as the modal density increases.

The analysis has been conducted first by means of the Galerkin technique, then by the asymptotic multiple scale method. which has permitted determination of the second-order differential equation that governs the amplitude modulation. As in parallel studies in a dynamic field. it is found that the phenomenon is described by a turning point problem which can be solved by the WKB method.

\section{REFERENCES}

Benito. R. and Sridharatn. S. (1985). Mode interiction in thin-walled structural members. J. Stract. Mech. 12(4). 517.542 .

Byskov. E. (1988). Elastic buckling problem with infinitely many lecal modes. Alech. Siruct. Wach. 15(4), 413 435 .

Byskov, E., Dambilde, L. and Jensen. K. J. (1989). Multimode interaction in axially stiffened cylindrical shells. Mech. Siruct. Hach $16(3), 387405$.

Cornwcll. P. J. and Hendiksen, O. O. (19k9). Leallization of vibrations in large space reflectors. Al.t.t J/ 27(2), $219 \cdot 226$.

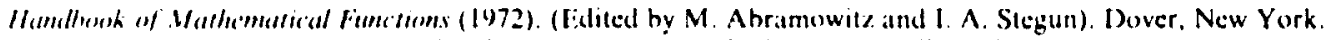

l.ungew. A. (198X). Mode lecilizalion hy structural imperfections in onc-dimensional continuous systems (int

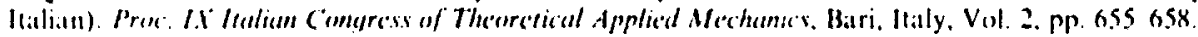

L.aongu, A and Pignataro, M. (I9Kא). Multiple interaction and loxalization phenomena in the posthuckling of compressed thin-walled members. AlA J/26(11). 1395 1402.

Moxham. K. E. (1971). Buckling lest on individual welded plates in compression. Cambridge University Engin. ecring Departmett, Repurt CUFD C.Structures/TR?

Nayleh. A. (1973). Merrurbation Me'fhods. John Wiley. Now York.

Perte, (and Cha, P. D. (I989). Strong mede locilization in nearly periodic disordered structures. AlA $J /$ 27(2). 227241.

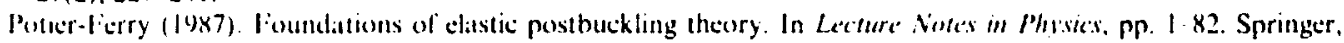
Herlin.

Sridharan, S. and Ali, A. (1985). Interattive buckling in thin-walled beam columns, ASCE 3(EM12), 1470-1486.

Trergatard, V. and Needlemin. A. (1980). On the locialization of buckling patterns. J. Appl. Mech. 47, 613-619. 